\title{
Do Chinese and British University Students Use Smartphones Differently? A Cross-cultural Mixed Methods Study
}

\author{
Zeyang Yang ${ }^{1}$ (D) $\cdot$ Kathryn Asbury ${ }^{1} \cdot$ Mark D. Griffiths $^{2}$
}

Published online: 6 November 2018

(C) The Author(s) 2018

\begin{abstract}
Although an increasing number of studies have focused on problematic smartphone use and smartphone addiction, few of these studies have employed both quantitative and qualitative methods or employed a cross-cultural design. A limited number of studies have compared eastern and western groups. The present study investigates the prevalence and causes of problematic smartphone use among Chinese and British undergraduates. A sample of $n=$ 778 undergraduates participated in this study (475 Chinese students and 303 British students). Students' scores on a self-report measure of problematic smartphone use were compared across country and gender. Qualitative data were analyzed using the framework approach. Chinese undergraduates reported significantly higher levels of PSU than British undergraduates, with a medium to large effect size. Females scored significantly higher than males in both groups. Chinese students reported that the sharp transition from a strictly managed high school life to a freer university life affected their level of smartphone use. This study indicates the importance of considering cultural and educational backgrounds when conducting studies on problematic smartphone use.
\end{abstract}

Keywords Problematic smartphone use $\cdot$ Smartphone addiction $\cdot$ Cross-cultural study $\cdot$ Mixed methods design $\cdot$ Student smartphone use

Smartphones, as mobile devices with flexible Internet access and diverse applications, are now widely used around the world. The global smartphone penetration rate has increased sharply in a short period, from 10\% in 2011 to $36 \%$ in 2018 (Statista 2018a). Research has found mobile phones (and more recently smartphones) can have both positive and negative impacts (Aoki

Zeyang Yang

zy664@york.ac.uk; yangzeyangyzy@hotmail.com

1 Psychology in Education Research Centre, Department of Education, University of York, York YO10 5DD, UK

2 International Gaming Research Unit, Psychology Department, Nottingham Trent University, Nottingham, UK 
and Downes 2003; Walsh et al. 2008). They provide highly convenient functions for daily social interaction and information searching but may also threaten individuals' privacy and their personal and financial safety (Aoki and Downes 2003). However, as discussed by Billieux (2012), in a minority of cases, problematic mobile phone use can also have harmful consequences. Studies across a range of countries have identified that problematic, excessive or addictive mobile phone (or smartphone) use can have negative psychological consequences such as anxiety and/or low psychological wellbeing (Bianchi and Phillips 2005; Billieux et al. 2015a; Ha et al. 2008; Hussain et al. 2017; Lepp et al. 2014; Leung 2008). It appears that the more negative side of smartphone use could be a global problem and more studies are needed to understand when, where, and how access to smartphones may be harmful.

Ever since the terms 'technological addiction' (Griffiths 1995) and 'internet addiction' (Griffiths 1996; Young 1998a, b) were proposed, many studies in the 2000s began to label problematic or dysfunctional smartphone use as an addiction (Kuss et al. 2014). Several theoretical frameworks focused on internet use have been proposed, including the cognitivebehavioral model of pathological internet use by Davis (2001) and a model of generalized internet addiction by Brand et al. (2014). However, the fifth edition of the Diagnostic and Statistical Manual of Mental Disorders (DSM-5; American Psychiatric Association 2013) does not contain any criteria for smartphone or internet addiction. Only Internet Gaming Disorder was included. Billieux et al. (2015b) express doubts about problematic mobile phone use being a behavioral addiction, while Griffiths $(2005,2017)$ argues that any behavior can be addictive if six key components of addiction are present (i.e., salience, conflict, tolerance, relapse, mood modification, and withdrawal symptoms). However, irrespective of whether such behavior should be classed as an 'addiction', as Bianchi and Phillips (2005) argued mobile phone use can be problematic and it is important to investigate problematic behavior regardless of labels. In the present study, the term 'problematic smartphone use' (PSU) is used given the ongoing debate concerning smartphone/mobile phone addiction. The term smartphone refers to mobile phones with Wi-Fi enabled functions and applications and flexible access to the Internet.

It appears that cultural contexts may affect, or be related to, patterns of smartphone use. Using the Smartphone Addiction Scale-Short Version (SAS-SV; Kwon et al. 2013), studies in diverse contexts reported different proportions of addicted smartphone users. The reported prevalence rates include Chinese students $(62.3 \%$ females, $64.9 \%$ males; Lachmann et al. 2018); Korean students (52\% females, $42 \%$ males; Kee et al. 2016); students from the UK comprising a mix of cultural and ethnic backgrounds but primarily British (32.3\% females, $29 \%$ males; Chotpitayasunondh and Douglas 2016); Belgian adults (22\% females, $20 \%$ males; Lopez-Fernandez 2017); Spanish adults (10.2\% females, $15.2 \%$ males; Lopez-Fernandez 2017); African American students (11.23\%; Lee 2015); and German students (8.7\% females, $6.2 \%$ males; Lachmann et al. 2018). It is notable that based on these reported studies, the prevalence of problematic smartphone use is highest among Asian samples. A recent crosscultural study conducted in Europe did not found a significant difference in PSU between Belgian and Finnish samples (Lopez-Fernandez et al. 2018).

China has been the world's largest smartphone market since 2012, and the number of mobile phone users is predicted to be 1.1 billion by 2019 with $63 \%$ of those being smartphone users (Statista 2018b). Chinese undergraduates have reported higher levels of problematic smartphone use than other Asian samples with similar cultural backgrounds (Long et al. 2016). Also, several recent Chinese studies have found college students' smartphone use to be associated with low psychological wellbeing, anxiety and loneliness (Bian and Leung 2015; Huang et al. 2013; Long et al. 2016). The situation appears to be similar in the UK. 
Smartphone ownership among British young people aged between 16 and 24 years was 96\% in 2017 , compared to $66 \%$ in 2012 (Statista 2018c). In a European cross-cultural study with 2775 young adults aged between 18 and 29 years, British young adults showed high levels of self-reported mobile phone dependence, ranking in second place, behind Belgium, among participants from nine European countries (Lopez-Fernandez et al. 2017). In another study, PSU was found to be associated with anxiety and low emotional stability among 640 participants in two British universities of whom the majority of participants (86\%) were British (Hussain et al. 2017).

More recently, Lachmann et al. (2018) reported that Chinese students scored higher on the SASSV than German students. However, the reason for this disparity remains unclear. The research so far suggests that problematic smartphone use is a particular problem in Chinese students, among eastern nations. There are also some signs that it may be more of a problem for British students than for students in other western nations, although this is less clear. It is not known why the prevalence of PSU is higher in these samples and no existing study has ever compared Chinese and British undergraduate students' PSU. It remains unclear whether different cultural backgrounds or educational contexts (e.g., schooling) makes a difference to students' self-control when using their smartphones. It also remains unclear whether the patterns, motivators, and impacts of PSU differ by cultural background. For instance, do Chinese and British people use their phones in the same way and for the same reasons? Moreover, to our knowledge, no study has investigated the prevalence, causes or solutions for PSU among university students across China and UK. Consequently, there is a good rationale to explore whether Chinese and British undergraduates perceive their smartphone use similarly or differently.

Most studies of PSU and mobile phone addiction use questionnaires to collect quantitative data, while only a limited number of qualitative studies have been conducted. To the present authors' knowledge, no existing study has ever explored and compared PSU using individuals from eastern/Asian and western/European countries, and collected both quantitative and qualitative data. Therefore, the present study explores whether there is a difference in selfreported PSU between Chinese and British undergraduates using a quantitative method and to gain some insight into what may underpin any observed differences via a qualitative method.

\section{Methods}

\section{Participants}

In the present study, there was a total of 778 participants (475 Chinese and 303 British undergraduates). Participants from China (266 males and 209 females) were Chinese undergraduates who were born and raised in mainland China. They were studying in a university in South China and represented those studying English, Computer Science, Management, Business, and Media. The average age was 19.8 years $(S D=1.11)$. Sixteen of these Chinese students volunteered to participate in follow-up interviews. The 303 British undergraduates (245 females, 57 males and 1 non-binary participant) were born and raised in the UK. All of the British participants were students at a university in northern England and represented a broad range of areas studying including Archeology, Economics, Education, Electronic Engineering, Health Science, Music, Philosophy, Psychology and Sociology. The average age of participants was 20.14 years $(S D=3.23)$ ranging from 18 to 50 years (with six participants aged over 30 years). 


\section{Measures}

PSU was assessed using the Smartphone Addiction Scale-Short Version (SAS-SV; Kwon et al. 2013). The SAS-SV comprises 10 items and participants respond to them using a 6-point Likert scale ranging from 'strongly disagree' (1) to 'strongly agree' (6). The Cronbach's alpha was 0.91 in the original validation study (Kwon et al. 2013). In the present study, it was 0.82 (Chinese $=0.80$ and British $=0.83$ ). A sample item is 'My smartphone is on my mind even when I am not using it'. Cut-off points for 'smartphone addiction' were provided by the developers: 33 points for females and 31 points for males (out of 60). For the Chinese students, the SAS-SV was translated into Chinese and 'Twitter and Facebook' were replaced by 'WeChat or other social media'.

For the Chinese students, an interview schedule was designed including six main questions: (1) Could you talk about the role of your smartphone in your daily life? (2) Could you describe how you use your smartphone in class or while you are studying? (3) Could you talk a bit about others' use of smartphones in their daily lives or during classes and other learning periods, from your point of view? (4) What factors do you think motivate you or your friends to use smartphones? (5) What are the possible impacts, if any, of smartphone use for you or your friends? (6) Is there anything you would like to add?

In the British sample, following administration of the SAS-SV, five open questions were included in the questionnaire to generate qualitative data. The questions were as follows: (1) Could you describe how you use your smartphone during class or other learning periods? (2) Could you talk a bit about the others' use of smartphones as you have observed it, either in their daily lives or during classes and other learning periods? (3) What factors motivate you or your friends to use smartphones? (4) What are the possible impacts, if any, of smartphone use to you or your friends? (5) Do you think you use your smartphone too much? If so, why? What problems does this cause for you? For practical reasons, British undergraduates were not interviewed and so qualitative data were gathered in different ways in each sample.

\section{Procedure}

The present study was designed as a cross-cultural mixed methods study which is helpful in reducing the limitations of both quantitative and qualitative data collection (Creswell and Plano Clark 2017; McMillan 2012). A qualitative design was included in the present study to help to acquire first-hand explanations of the SAS-SV scores and correlates of PSU. Both paper and online questionnaires were used to recruit as many participants as possible. The survey was printed on folded A3 sheets for offline distribution. In both China and the UK, the surveys were distributed around the university campus and during breaks in lectures, where staff permission had been given. As part of the paper-based data-collection process the first author introduced the study to potential participants before distributing the paper surveys. The students read the informed consent sheet before deciding whether to participate.

At the end of the Chinese questionnaires, participants were asked to give their contact number if they would like to be interviewed and 16 Chinese students agreed to take part in semi-structured interviews. Interviews were conducted in Mandarin and were recorded with participants' permission. In order to generate qualitative data that might shed light on or explain participants' survey responses, five open, free-response, items were added for the British students (see above for details). 


\section{Data Analysis}

The total scores for the SAS-SV in the Chinese and British samples were calculated and compared. The percentages of 'addicted' users (using the thresholds described by the instrument developers) were compared between the two groups. Independent samples t-tests were used to test for gender differences and for differences in SAS-SV scores between the two groups. The framework analytic approach (Ritchie and Spencer 1994) was used for qualitative data analysis. It is a widely used approach for qualitative data analysis (Gale et al. 2013). This approach includes five stages: (1) familiarization, (2) identifying a thematic framework, (3) indexing, (4) charting, (5) mapping and interpretation (Ritchie and Spencer 1994). Data are reduced and organized into a table in which rows represent cases and columns represent themes. As a systematic method, the framework approach can be used deductively, inductively or in a combined way (Gale et al. 2013). For the Chinese students' interviews, transcriptions were directly coded in Chinese in order to avoid information loss in the process of translation. Coding for the Chinese interview transcripts and British students' narrative responses was conducted in NVivo version 11. Deductive coding was carried out according to the research aims. Therefore, the codes were categorized as follows: the levels of PSU; the antecedents and consequences of PSU. Meanwhile, inductive coding was conducted to find out whether there are other predictors, consequences or any other factors related to PSU.

\section{Results}

\section{Quantitative Findings}

Table 1 shows descriptive statistics for the SAS-SV scores of both Chinese and British students. The observed ranges indicate that the responses were sufficiently well distributed. Skewness and Kurtosis values show that all data were normally distributed. Cronbach's alpha values indicate that the SAS-SV obtained excellent reliabilities among both groups. The medians of corrected item-total correlations also suggested good internal consistency with all values above 49 .

Independent samples $t$ tests showed that there were significant gender differences for both Chinese and British undergraduates. As shown in Table 2, females reported significantly higher scores on the SAS-SV than males (Chinese: $t(473)=3.07, p<.01$; British: $t(300)=$ $2.80, p<.01)$. The effect size of gender difference was larger in the British sample $(d=-.41)$ than the Chinese sample $(d=-.28)$, though both the effect sizes are small according to the criteria in Cohen (1988). Table 3 shows a comparison of the percentage of participants who

Table 1 Descriptive statistics for the SAS-SV scores in Chinese and British students

\begin{tabular}{|c|c|c|c|c|c|c|c|c|c|c|}
\hline \multirow[t]{2}{*}{ Country } & \multirow[t]{2}{*}{$\mathrm{N}$} & \multirow[t]{2}{*}{ No. of items } & \multicolumn{2}{|l|}{ Range } & \multirow[t]{2}{*}{$M$} & \multirow[t]{2}{*}{$S D$} & \multirow[t]{2}{*}{ Skewness } & \multirow[t]{2}{*}{ Kurtosis } & \multirow[t]{2}{*}{$\mathrm{M}^{\mathrm{R}}$} & \multirow[t]{2}{*}{$\alpha$} \\
\hline & & & Potential & Observed & & & & & & \\
\hline China & 475 & 10 & $10-60$ & $10-60$ & 36.70 & 7.55 & .05 & .31 & .49 & .80 \\
\hline UK & 303 & 10 & $10-60$ & $12-56$ & 31.96 & 8.57 & .24 & -.04 & .53 & .83 \\
\hline
\end{tabular}

Note. $\mathrm{M}^{\mathrm{R}}=$ Median of corrected item-total correlations 
Table 2 Gender differences for SAS-SV scores among Chinese and British undergraduates

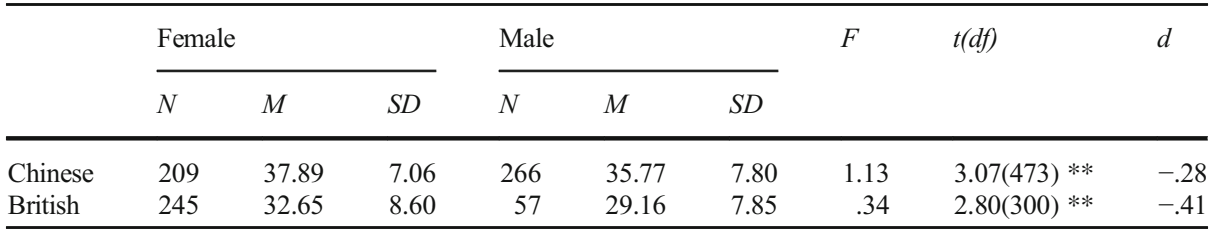

Note. $* * p<0.01$

scored above the threshold of 'smartphone addiction' set out in the SAS-SV. Both females and males in the British sample showed lower levels of 'smartphone addiction' than Chinese students (see Table 2).

British students reported less problematic smartphone use. They scored significantly lower in the SAS-SV than the Chinese sample with a large effect size $(t[583.86]=7.89, p<.001, d=$ - .60). This is in accordance with the lower percentages of 'addicted' smartphone users observed in the British sample (40-43\%) compared with $72-73 \%$ in the Chinese sample.

\section{Qualitative Findings}

\section{Similar Patterns of PSU}

Chinese and British undergraduates reported similar patterns of PSU as described in the model of problematic mobile phone use in Billieux et al. (2015a): concerning the addictive pattern, antisocial pattern and risky pattern. Evidence for all three patterns of use was confirmed. In terms of the addictive pattern, participants explicitly used words such as 'addiction' or 'addictive', and reported frequent checking of their phones or using smartphones late at night.

I saw some classmates playing games during the whole class...Their gaming [via smartphone] is a kind of addiction (Chinese female, 20, SAS-SV score: 27).

Nowadays many people have a kind of psychological addiction. It's just like smoking addiction. They might feel someone was contacting them when they don't have a smartphone at hand (Chinese male, 20, SAS-SV score: 33).

It is addictive and hard to tune out, and often sleep [because your] head is filled with phone stuff (British female, 19, SAS-SV score: 44).

People can get addicted to the instant-gratification kick that smartphones offer. Perpetually checking social media and various websites, scrolling non-stop... Smartphones are second-to-none when it comes to wasting time (British female, 22, SAS-SV score: 23)

Table 3 Comparison of the SAS-SV scores between Chinese and British samples

\begin{tabular}{lllllllll}
\hline & $N$ & Mean & $S D$ & $d$ & $F$ & $t(d f)$ & & \multicolumn{2}{l}{ 'Addicted' (over threshold) } \\
\cline { 3 - 7 } & & & & & & Male $>31$ & Female $>33$ \\
\hline Chinese & 475 & 36.70 & 7.55 & -0.60 & $4.12^{*}$ & $7.89(583.86)^{* * *}$ & $193 / 266(72.56 \%)$ & $151 / 209(72.25 \%)$ \\
British & 303 & 31.96 & 8.57 & & & & $23 / 57(40.35 \%)$ & $106 / 245(43.27 \%)$ \\
\hline
\end{tabular}

Note. $* p<0.05$. $* * * p<.001$ 
The participants also reported antisocial patterns including irrelevant use in classes and social gatherings, as well as examples of dangerous use:

Everyone is using smartphones. Now when friends meet in a party, they sit down and just use smartphones...I think we'd better not have parties. Just chat online...It's always the case recently (Chinese female, 20, SAS-SV score: 50)

Antisocial periods, we don't sit and properly watch a film, we all sit on our phones (British female, 21, SAS-SV score: 25)

To escape (through apps but mostly listening to music), as a social crutch (British, male, 21, SAS-SV score: 42).

Sometimes though, I see people using their phones while in class or lectures or excessively at dinner with friends and it surprises me (British female, 20, SAS-SV score: 40$)$.

Not taking care when walking (British Female, 50, SAS-SV score: 40)

A number of British participants reported that they rarely used their smartphones during lectures. However, as was also true in China, their words were not consistent with their selfperceived PSU. A few of them reported behavior that was inconsistent with their SAS-SV scores. For example:

I don't think I use my smartphone too much. I use it less than the others around me (Chinese male, 20, SAS-SV score: 33).

I do not tend to use my phone in class because my attention is held a lot more in these situations - it is when I have to rely on my self-discipline that the problems occur (British male, 18, SAS-SV score: 49).

One British participant specified Chinese students in particular as heavy users, according to her own observation.

People spend a lot of time on social media during times when they should be paying attention - particularly Chinese students (British female, 19, SAS-SV score: 26).

The study also explored what students in both samples perceived as the precursors to, and consequences of, problematic smartphone use and similarities were observed here too.

\section{Similar Antecedents for PSU}

British undergraduates reported similar antecedents for PSU as Chinese students including internal reasons (e.g., fear of missing out on something, need for reassurance, poor selfregulation) and external reasons (e.g., boring lecture, peer effect).

There are some reasons of myself. I'm not that kind of person who can sit in the classroom and concentrate on the teacher all the time. Sometimes I distract from the class and take out my smartphone for leisure (Chinese male, 18, SAS-SV score: 39) My self-control is poor. I used my smartphone for leisure when the class was so boring that I couldn't focus on it any longer...I think the reason is me myself. I didn't understand the course so I couldn't control myself and stop myself from using my smartphone (Chinese female, 19, SAS-SV score: 48)

I don't use my phone in class, but when studying I go on Facebook and get trapped scrolling through the news feed (British female, 19, SAS-SV score: 36) 
[I don't use my smartphone] at all during lectures) if the lecture is interesting, browse social media and message friends if the lecture boring (British female, 26, SAS-SV score: 25 )

When I am surrounded people who are also procrastinating, I find it easy to do myself without feeling guilty (British female, 22, SAS-SV score: 36 )

\section{Similar Impacts}

As reported by one British participant, checking smartphones is not always problematic and it depends on the impact of the behavior.

Some of my peers will be constantly on their phone, trying to hide it from the lecturer. Others will put their phone in their bag and not touch it throughout the entire lecture, though this might be rare among my age group. I think it would be unusual now for a student to go more than one or two hours without at least checking their phone for the time or notifications. Even one of my lecturers always uses his phone to check the time. I don't have a problem with this as long as it does not distract from the task at hand (British female, 21, SAS-SV score: 27).

Chinese and British students reported both positive and negative impacts of smartphones, including positives such as convenience, help with study and reassurance. For instance:

I think smartphone is more positive to my life. I have many feelings to relieve...I relieve my feeling by reading or listening to music at that time. But now I played games once I had some negative emotions. Now, if I am very angry or tense, I would think about my smartphone and play games (Chinese male, 18, SAS-SV score: 30)

I can watch some TED videos. They have many new thoughts. A lot of presentations. Really useful (Chinese male, 19, SAS-SV score: 43)

I usually use it for looking things up and that I don't understand, which relates to what we are learning about (British female, 20, SAS-SV score: 26).

Practicality. The minor things, like being able to check a map and travel apps (I grew up in London, Citymapper is a godsend). I'm not into social media, but being able to message people on Facebook is admittedly useful. Keeping track of eBay, listening to music, checking the news. Smartphones can help make the day-to-day more streamlined (British female, 22, SAS-SV score: 23).

It's also how I wind down or relax, as it's not a stressful activity (British female, 19, SAS-SV score: 39 ).

However, negative impacts of smartphones were also reported including poorer social relationships, poorer physical health, distraction, time wasting, procrastination and anxiety.

It negatively affected my eyesight and my neck (Chinese female, 20, SAS-SV score: 27).

It takes too much time in our lives. Most time was used on smartphones. I feel I should have done many things but I spent the time on my smartphone (Chinese female, 19, SAS-SV score: 48 ).

Staying up too late and being tired the next day (British female, 18, SAS-SV score: 47). It distracts me a lot from work, which causes me to be more stressed in the long run (British female, 19, SAS-SV score: 39). 
I spend too much time on social media (comment sections are toxic but addictive), and I sometimes spend too much time on smartphone games. This causes some issues as I end up putting off work or chores I planned to get on with (British female, 20, SAS-SV score: 35$)$.

\section{Similar Perceptions}

Both Chinese and British students reported a perception that they needed to change their ways of smartphone use by reducing the amount of time they spend on them. Those who showed their awareness of their overuse of smartphones tended to be low scoring on the SAS-SV.

As a university student, I need to read more books and use smartphone less (Chinese female, 18, SAS-SV score: 42).

I don't even use it that much. But I still think, if I used the time that I spent flicking through the news (or even Buzzfeed, god forbid) to read a book, then that would have been time well spent (British female, 22, SAS-SV score: 23).

Not necessarily problems in my life but I mean, when asking the question what could I have done better, there is always at least something I could have done rather than be on a phone (British male, 20, SAS-SV score: 20)

\section{Differences}

There were also several differences observed in the qualitative responses from the Chinese and British samples. First, the Chinese sample reported using more types of functions and more specific smartphone applications than British students did. Chinese students mentioned not only social media and information searching tools, but also applications for mobile payment, sports and exercise, shared bikes, leisure reading, word memorizing tools, online courses, offclass tests, and university required applications. The majority of the British students reported the same functions including social media (Facebook, Instagram, Snapchat) and information searching. It appears from these data that the Chinese students used their smartphones for more things, possibly explaining their higher level of usage to some extent. The Chinese students mentioned threats to privacy while British students did not mention this as a problem. This may also indicate that the Chinese students perceived themselves as living in a more complex context relying on a more diverse range of smartphone functions.

Second, British students did not mention the effect of transition from high school to university. Where Chinese students complained about their highly restricted use of smartphones in high school, British students did not mention any changes between high school and university life. The sharp contrast between high school and university lifestyle in relation to use of smartphones appears only applicable to the Chinese students. This appears to be a key difference between the two groups.

My high school has very strict discipline. After enrolling in university, I suddenly found that nobody is controlling us anymore. We were frequently told by high school teachers that we would be free and relaxed once we go to the university...In high school, I didn't have my own smartphone, I used my parents' phones for fun on weekends (Chinese male, 19, SAS-SV score: 32) 
In high school, I never used my smartphone during class or in individual learning periods. But in university, it's different. We didn't have much time in high school. We needed to take part in many extracurricular activities. Academic pressure is not that high in university (Chinese female, 20, SAS-SV score: 34)

One British student reported that smartphones are not the only distractor since they may turn to the other devices such as laptops during lectures for irrelevant use. It is important to note that smartphones are not the only devices that can distract students from their tasks. When students cannot control themselves well, they may lose concentration and use smartphones, laptops or other devices for distraction.

In class I see a lot of people using laptops to check social media - this then looks to the teachers like they are taking notes. However, this seems to be a way of accessing smartphone features/apps through another device - if it were allowed/not considered rude to be on smartphones in class these would probably be used instead. In terms of daily lives, I rarely seem to see people glued to their phone when they are in social groups, but when they are alone in a public setting people (perhaps under the age of 40) will be on their smartphone (British female, 21, SAS-SV score: 30 ).

\section{Discussion}

Compared with Chinese undergraduates, British undergraduates reported lower levels of PSU. However, the rates were still relatively high compared to other countries, as shown in Table 4. Females' smartphone use tended to be more problematic than males in both China and the UK. The qualitative data indicated that British students had similar views of the patterns, antecedents, and impacts of PSU as their Chinese counterparts. However, Chinese students appeared to experience a different context in which they perceived smartphones to have more wideranging functions and reported accessing more applications than the British students. The sharp contrast between high school and university life appeared to be another specific problem for Chinese students, which was not mentioned at all by the British students.

Table 4 Cross-cultural comparison of the percentages of addicted smartphone users judged by the thresholds of the SAS-SV

\begin{tabular}{lllr}
\hline & Female & Male & Total \\
\hline African American (>35) & - & - & $11.23 \%$ \\
Belgian (>32) & $22 \%$ & $20 \%$ & $21.52 \%$ \\
Chinese in Lachmann et al. (2018) & $62.3 \%$ & $64.9 \%$ & $64.22 \%$ \\
German & $8.7 \%$ & $6.2 \%$ & $7.9 \%$ \\
Korean & $52 \%$ & $42 \%$ & $50 \%$ \\
Multi-national & $32.3 \%$ & $29 \%$ & $31.08 \%$ \\
Spanish (>32) & $10.2 \%$ & $15.2 \%$ & $12.82 \%$ \\
British students in the current study & $43.27 \%$ & $40.35 \%$ & $42.72 \%$ \\
Chinese students in the current study & $72.25 \%$ & $72.56 \%$ & $72.42 \%$ \\
\hline
\end{tabular}

Note. Cut-off points were changed to 35 for the African American sample by Lee (2015), and 32 for Belgian and Spanish samples in Lopez-Fernandez (2017). The Korean sample is from Kee et al. (2016), German sample is from Lachmann et al. (2018), and the multinational sample (mainly British) is from Chotpitayasunondh and Douglas (2016) 
This study has several empirical and methodological implications. First, it is important to consider cultural backgrounds and educational experience when investigating PSU. Second, the reliability of the thresholds for PSU or smartphone addiction in self-reported scales requires more discussion and investigation. Furthermore, it appears that PSU could be considered as both generalized PSU and specific PSU.

In line with a cross-cultural study of Chinese and German students (Lachmann et al. 2018), the present study found Chinese undergraduates reported higher levels of PSU than British students. It appears there is a cultural difference regarding PSU between Chinese and European samples. As shown in Table 4, the Chinese and British samples in this study both showed high levels of smartphone addiction using the thresholds provided by the developers of the SAS-SV, but Chinese scores were significantly higher. The Chinese sample studied by Lachmann et al. (2018) also scored higher than samples from other countries. This situation might be explained by Chinese students' difficulties with the sharp transition from highly stressful and externally regulated high school life to a much more relaxed and free university life, a situation that was only reported by the Chinese students in the present study. It indicates that cultural backgrounds and previous educational experiences might be influential factors to consider when studying college students' use of technology (including smartphones).

Cultural differences also appear to exist between Eastern and Western countries since both Chinese and Korean samples scored higher than non-Asian samples in previous studies (Kee et al. 2016; Lachmann et al. 2018; Lee 2015; Lopez-Fernandez 2017). The British sample in the current study - similar to the multicultural sample (mainly British) in the study by Chotpitayasunondh and Douglas (2016) — scored higher rates of PSU than other samples from Europe and America. This again appears to indicate the importance of cultural difference.

The very high levels of PSU in Chinese students, and the relatively high levels in British students, are potentially one of concern. However, it should also be noted that the SAS-SV has arguably low cut-off points ( 31 and 33 out of 60) at around the mid-point of the scale. It may be that scores around the mid-point of the scale do not discriminate excessive and preoccupied smartphone users from those 'addicted'. Evidence from the present study appears to support this because for several participants, in both the Chinese and British samples, qualitative accounts of smartphone use were not well aligned with their scores for the SAS-SV. It is important to discuss the reliability and validity of self-reported scales for smartphone addiction and PSU such as the SAS-SV. Another factor to consider is that there may be a cultural difference in how smartphone use is perceived in eastern and western cultures. Griffiths et al. (2016) reported that parents in South-East Asian cultures tend to pathologize any activity that takes away time from education and the family. Therefore, any smartphone use may be viewed as potentially problematic in countries such as China, even if the usage is 'normal'.

It appears possible to classify PSU as generalized PSU and specific PSU, similar to the classification of generalized and specific pathological Internet Use (Davis 2001) or Internet addiction (Brand et al. (2014). Both British and Chinese participants mentioned the problematic use of specific functions of smartphone (e.g., Facebook), and generalized antisocial use at gatherings or parties. In some cases, PSU can reflect other factors including social anxiety and poor self-regulation. Meanwhile, for some students, the specific functions or applications found on smartphones appear to underlie PSU. As mentioned by one British participant, as well as smartphones, students can be distracted by the other devices with similar functions, such as laptops. As reported by both the Chinese and British students, some smartphone functions can be beneficial to their life and study. It indicates the importance of considering the specific functions/usage of smartphones when designing the scales for PSU and smartphone 
addiction. A recent validated Smartphone Application-Based Addiction Scale (SABAS; Csibi et al. 2018) based on the six key components of addiction (Griffiths 2005, 2017) might be a good example of developing functional specific scales for PSU or smartphone addiction.

One limitation of the research presented here might be the different methods of qualitative data collection from the Chinese and British students, although interesting similarities and differences were identified. As the scores on the scale used to assess PSU and the words of the participants were not always consistent, it appears that the participants may have given socially desirable answers and/or the scale's cut-off in assessing PSU was too low. Another limitation that all the data were self-report and this form of data collection suffers from well-known biases that could have affected the findings (e.g., selection biases, memory recall biases, social desirability biases, etc.). In addition, the sample size that lacks representativeness could be a limitation.

For future studies on this topic, cross-cultural research appears to be an important line of enquiry given that the study identified significant cultural differences between Chinese and British university students, and supported findings from extant research. More mixed methods and longitudinal studies are needed, especially using and comparing both Eastern and Western samples. Furthermore, the development of functional-specific scales with greater validity will be important new direction for scale development for the field of smartphone addiction and PSU. Finally, a significant gender difference in PSU was noted in the present study but the qualitative data did not shed any light on the causes of this. It may be that given there is an association between smartphone addiction and social media addiction (Kuss and Griffiths 2017), and that females tend to be more sociable than males, it may be that females use smartphones more than males for its social benefits and easy access to social media. Also, largescale studies have demonstrated that females tend to spend more time and are more addicted to social media than males whereas males spend more time gaming and are more addicted to gaming than females (e.g., Andreassen et al. 2016). Given that smartphones are not a good medium for playing massively multiplayer games (Lopez-Fernandez et al. 2018), males are more likely to spending excessive amounts of times on laptops and personal computers pursuing their favorite leisure activities than females. This also represents another good line of inquiry for future research.

Authors' Contribution Zeyang Yang and Kathryn Asbury designed the study. Zeyang Yang conducted data collection and analysis. Zeyang Yang carried out the main bulk of the manuscript writing and literature review. Kathryn Asbury participated in checking methods and results and supporting Zeyang Yang during the data collection and analysis. Kathryn Asbury and Mark D. Griffiths acted in an editorial role when it came to the writing up of the research study. All authors read and approved the final manuscript.

\section{Compliance with Ethical Standards}

Conflict of Interest The authors declare that they have no conflict of interest.

Ethics This study has been approved by the Ethics Committee of the Department of Education at the University of York.

Open Access This article is distributed under the terms of the Creative Commons Attribution 4.0 International License (http://creativecommons.org/licenses/by/4.0/), which permits unrestricted use, distribution, and reproduction in any medium, provided you give appropriate credit to the original author(s) and the source, provide a link to the Creative Commons license, and indicate if changes were made. 


\section{References}

American Psychiatric Association. (2013). Diagnostic and statistical manual of mental disorders. Arlington, VA: American Psychiatric Association.

Andreassen, C. S., Billieux, J., Griffiths, M. D., Kuss, D. J., Demetrovics, Z., Mazzoni, E., \& Pallesen, S. (2016). The relationship between addictive use of social media and video games and symptoms of psychiatric disorders: A large-scale cross-sectional study. Psychology of Addictive Behaviors, 30, 252-262.

Aoki, K., \& Downes, E. J. (2003). An analysis of young people's use of and attitudes toward cell phones. Telematics and Informatics, 20, 349-364.

Bian, M., \& Leung, L. (2015). Linking loneliness, shyness, smartphone addiction symptoms, and patterns of smartphone use to social capital. Social Science Computer Review, 33(1), 61-79.

Bianchi, A., \& Phillips, J. G. (2005). Psychological predictors of problem mobile phone use. Cyberpsychology \& Behavior, 8(1), 39-51.

Billieux, J. (2012). Problematic use of the mobile phone: A literature review and a pathways model. Current Psychiatry Reviews, 8(4), 299-307.

Billieux, J., Maurage, P., Lopez-Fernandez, O., Kuss, D. J., \& Griffiths, M. D. (2015a). Can disordered mobile phone use be considered a behavioral addiction? An update on current evidence and a comprehensive model for future research. Current Addiction Reports, 2(2), 156-162.

Billieux, J., Philippot, P., Schmid, C., Maurage, P., De Mol, J., \& Van der Linden, M. (2015b). Is dysfunctional use of the mobile phone a behavioural addiction? Confronting symptom-based versus process-based approaches. Clinical Psychology \& Psychotherapy, 22(5), 460-468.

Brand, M., Laier, C., \& Young, K. S. (2014). Internet addiction: Coping styles, expectancies, and treatment implications. Frontiers in Psychology, 5, 1256.

Chotpitayasunondh, V., \& Douglas, K. M. (2016). How "phubbing" becomes the norm: The antecedents and consequences of snubbing via smartphone. Computers in Human Behavior, 63, 9-18.

Cohen, J. (1988). Statistical Power Analysis for the Behavioral Sciences (2nd ed.). Hillsdale, N.J.: L. Erlbaum Associates.

Creswell, J. W., \& Plano Clark, V. L. (2017). Designing and conducting mixed methods research (third ed.). Los Angeles: Sage.

Csibi, S., Griffiths, M. D., Cook, B., Demetrovics, Z., \& Szabo, A. (2018). The psychometric properties of the Smartphone Application-Based Addiction Scale (SABAS). International Journal of Mental Health and Addiction, 16(2), 393-403.

Davis, R. A. (2001). A cognitive-behavioral model of pathological Internet use. Computers in Human Behavior, 17(2), 187-195.

Gale, N. K., Heath, G., Cameron, E., Rashid, S., \& Redwood, S. (2013). Using the framework method for the analysis of qualitative data in multi-disciplinary health research. BMC Medical Research Methodology, 13, 117.

Griffiths, M. D. (1995). Technological addictions. Clinical Psychology Forum, 76, 14-19.

Griffiths, M. D. (1996). Internet addiction: an issue for clinical psychology? Clinical Psychology Forum, 97, 3236.

Griffiths, M. D. (2005). A 'components' model of addiction within a biopsychosocial framework. Journal of Substance Use, 10, 191-197.

Griffiths, M. D. (2017). Behavioural addiction and substance addiction should be defined by their similarities not their dissimilarities. Addiction, 112, 1718-1720.

Griffiths, M. D., Kuss, D. J., Billieux, J., \& Pontes, H. M. (2016). The evolution of internet addiction: A global perspective. Addictive Behaviors, 53, 193-195.

Ha, J. H., Chin, B., Park, D.-H., Ryu, S.-H., \& Yu, J. (2008). Characteristics of excessive cellular phone use in Korean adolescents. Cyberpsychology \& Behavior, 11(6), 783-784.

Huang, H., Zhou, C., \& Yu, L. (2013). Association between mobile phone addiction and mental health among college students. Chinese Journal of School Health, 34(9), 1074-1076.

Hussain, Z., Griffiths, M. D., \& Sheffield, D. (2017). An investigation in to problematic smartphone use: the role of narcissism, anxiety, and personality factors. Journal of Behavioral Addictions, 6, 378-386.

Kee, I. K., Byun, J. S., Jung, J. K., \& Choi, J. K. (2016). The presence of altered craniocervical posture and mobility in smartphone-addicted teenagers with temporomandibular disorders. Journal of Physical Therapy Science, 28, 339-346.

Kuss, D. J., \& Griffiths, M. D. (2017). Social networking sites and addiction: Ten lessons learned. International Journal of Environmental Research and Public Health, 14, 311.

Kuss, D. J., Griffiths, M. D., Karila, L., \& Billieux, J. (2014). Internet addiction: a systematic review of epidemiological research for the last decade. Current Pharmaceutical Design, 20, 4026-4052.

Kwon, M., Kim, D. J., Cho, H., \& Yang, S. (2013). The smartphone addiction scale: development and validation of a short version for adolescents. PLoS One, 8(12), e83558. 
Lachmann, B., Sindermann, C., Sariyska, R. Y., Luo, R., Melchers, M. C., Becker, B., Cooper, A. J., \& Montag, C. (2018). The role of empathy and life satisfaction in internet and smartphone use disorder. Frontiers in Psychology, 9, 398.

Lee, E. B. (2015). Too much information: Heavy smartphone and Facebook utilization by African American young adults. Journal of Black Studies, 46(1), 44-61.

Lepp, A., Barkley, J. E., \& Karpinski, A. C. (2014). The relationship between cell phone use, academic performance, anxiety, and satisfaction with life in college students. Computers in Human Behavior, 31(1), $343-350$.

Leung, L. (2008). Linking psychological attributes to addiction and improper use of the mobile phone among adolescents in Hong Kong. Journal of Children and Media, 2(2), 93-113.

Long, J., Liu, T. Q., Liao, Y. H., Qi, C., He, H. Y., Chen, S. B., \& Billieux, J. (2016). Prevalence and correlates of problematic smartphone use in a large random sample of Chinese undergraduates. BMC Psychiatry, 16, 408.

Lopez-Fernandez, O. (2017). Short version of the Smartphone Addiction Scale adapted to Spanish and French: Towards a cross-cultural research in problematic mobile phone use. Addictive Behaviors, 64, 275-280.

Lopez-Fernandez, O., Kuss, D. J., Romo, L., Morvan, Y., Kern, L., Graziani, P., Rousseau, A., Rumpf, H. J., Bischof, A., Gässler, A. K., Schimmenti, A., Passanisi, A., Männikkö, N., Kääriänen, M., Demetrovics, Z., Király, O., Chóliz, M., Zacarés, J. J., Serra, E., Griffiths, M. D., Pontes, H. M., Lelonek-Kuleta, B., Chwaszcz, J., Zullino, D., Rochat, L., Achab, S., \& Billieux, J. (2017). Self-reported dependence on mobile phones in young adults: A European cross-cultural empirical survey. Journal of Behavioral Addictions, 6 , $168-177$.

Lopez-Fernandez, O., Männikkö, N., Kääriäinen, M., Griffiths, M. D., \& Kuss, D. J. (2018). Mobile gaming and problematic smartphone use: A comparative study between Belgium and Finland. Journal of Behavioral Addictions, 7(1), 88-99.

McMillan, J. H. (2012). Educational research: Fundamentals for the consumer (6th ed.). Boston: Pearson.

Ritchie, J., \& Spencer, L. (1994). Qualitative data analysis for applied policy research. In A. Bryman \& R. Burgess (Eds.), Analysing qualitative data (pp. 173-194). London: Routledge.

Statista (2018a). Number of smartphone users worldwide from 2014 to 2020 (in billions). Retrieved June 14, 2018, from: https:/www.statista.com/statistics/330695/number-of-smartphone-users-worldwide/

Statista (2018b). China: Smartphone Market - Statistics \& Facts. Retrieved June 14, 2018, from: https://www. statista.com/topics/1416/smartphone-market-in-china/

Statista (2018c). UK: smartphone ownership by age from 2012-2017. Retrieved June 14, 2018, from https://www.statista.com/statistics/271851/smartphone-owners-in-the-united-kingdom-uk-by-age/.

Walsh, S. P., White, K. M., \& Young, R. M. (2008). Over-connected? A qualitative exploration of the relationship between Australian youth and their mobile phones. Journal of Adolescence, 31(1), 77-92.

Young, K. S. (1998a). Caught in the net: How to recognize the signs of internet addiction and a winning strategy for recovery. New York: Wiley.

Young, K. S. (1998b). Internet addiction: The emergence of a new clinical disorder. Cyberpsychology \& Behavior, 1(3), 237-244. 